\title{
A LARGE-APERTURE NARROW QUADRUPOLE FOR THE SNS ACCUMULATOR RING*
}

\begin{abstract}
N. Tsoupas, J. Brodowski, W. Meng, J. Wei, Y.Y. Lee, J. Tuozzolo, BNL, Upton, NY 11973 USA Abstract

The accumulator ring of the Spallation Neutron Source (SNS) [1] is designed to accept high-intensity $\mathrm{H}$ beam of $1 \mathrm{GeV}$ kinetic energy from the injecting LINAC, and to accumulate, in a time interval of $1 \mathrm{msec}, 2 \times 10^{14}$ protons in a single bunch of 700 nsec. In order to optimize the effective straight-section spaces for beaminjection[2], extraction[3] and collimation[4], we have minimized the width of the large aperture quadrupoles which are located in the same straight sections of the accumulator ring with the injection and extraction systems. By minimizing the width of the quadrupoles to

The size of $\left(\mathrm{x}_{\mathrm{cod}}\right)$ of the local bump can be reduced by moving the injection septum magnet closer to the ring by reducing the transverse dimensions $\left(\mathrm{W}_{\text {quand }}\right)$ of the quadrupoles which otherwise would interfere. Similarly the quadrupole doublets which are located at the straight section where the beam-extraction system is located, interfere laterally with the extracted beam as shown in Fig. 1. Reduction of the quadrupole width $\left(\mathrm{W}_{\text {quad }}\right)$ reduces the beam extraction angle and allows lower B-field of the septum magnet, thus minimizing the effect of the septum magnet's fringing fields on the circulating beam.
\end{abstract} $\pm 40.4 \mathrm{~cm}$, the beam-injection and extraction angles are lowered to $8.75^{\circ}$ and $16.8^{\circ}$ respectively. Further optimization of the narrow quadrupole, minimizes the strength of the dodecapole multipole component of the quadrupole, thus reducing the width of the 12pole structure resonance and allowing a larger tune space for stability of the circulating beam[5]. In this paper we present results derived from magnetic field calculations of 2D and 3D modeling, and discuss the method of optimizing the size of the quadrupole and minimizing its dodecapole multipole component.

\section{WHY USE NARROW QUADRUPOLE?}

The SNS accumulator ring[1], schematically shown in Fig.1, is $248 \mathrm{~m}$ in length and consists of four identical arcs of $90^{\circ}$ each. The arcs are connected by four straight sections, each $31.5 \mathrm{~m}$ in length. As part of the ring's beam optics a set of two quadrupole-doublets are located along each of the straight sections (see Fig. 1), and symmetrically placed with respect to the center of each straight section. The presence of the quadrupole doublets in the straight sections of the ring where the beam-injection and beam-extraction systems are located (see Fig. 1), suggests that the dimensions of the quadrupoles determine the various parameters of the magnets associated with the beam-injection and extraction systems. A more detailed view of the injection region in Fig. 2, shows the quadrupole doublets, the injection septum, and the four chicane dipole magnets which generate the local orbit bump[2] necessary to displace the closed orbit at the location of the stripping foil. In order to minimize the magnetic-field-stripping of the $\mathrm{H}^{-}$beam and the $\mathrm{H}^{0}$ beam ${ }^{1}$ [7], the chicane magnets should be excited at the lowest possible magnetic fields. Low excitation fields of the chicane magnets requires smaller size of the local orbit bump $\left(\mathrm{x}_{\mathrm{cod}}\right)$ shown in Fig. 1.

* SNS is managed by UT-Battelle, LLC, under contract

DE-AC05-00OR22725 for the U.S. Department of Energy.

'The $\mathrm{H}^{0}$ beamis produçed by the incomplete stripping of the $\mathrm{H}$ beam by the stripping foil.

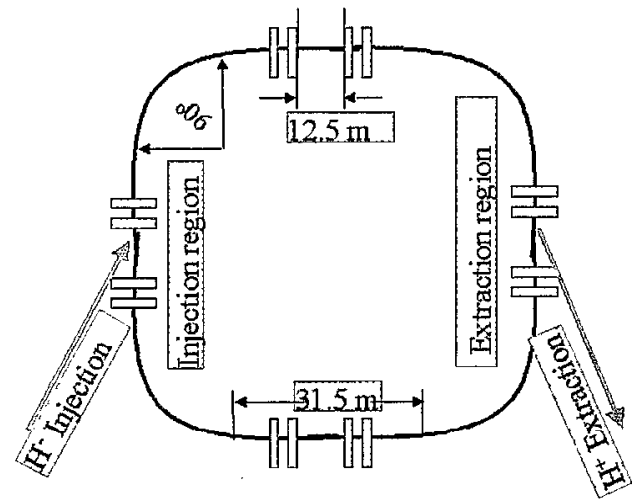

Figure1:Schematic diagram of the SNS accumulator ring.

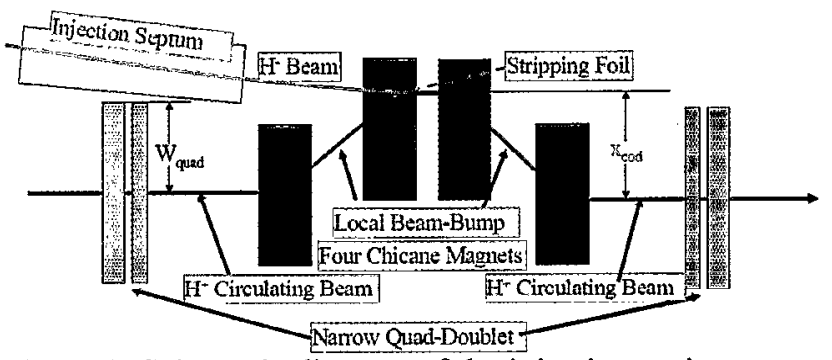

Figure 2: Schematic diagram of the injection region.

\section{REQUIREMENTS OF THE NARROW QUAD}

The cross-section of the narrow quadrupole is shown in Fig. 3. (iron $\Leftrightarrow$ blue, coils $\Leftrightarrow$ red). The dimensions of the narrow quadrupole magnets, which appear in Table 1 , were dctermined from a) the size of the beam at the location of the quadrupoles, b) the allowed uncontrolled beam-losses along the ring[4] and c) the magnetic-field requirements of the quadrupoles. The length, the width, and the pole-width (columns 3,4,5 Table 1) of the quad have been chosen to minimize the effects of the magnetic field saturation on the magnet and thus maintain the same field quality for 1.0 and $1.3 \mathrm{GeV}$ operations. The transverse width of $\pm 40.4 \mathrm{~cm}$ of the quadrupoles allows for $8.75^{\circ}$ and $16.8^{\circ}$ beam-injection and extraction angles. The fringe field of the quadrupole at $x<-40.4 \mathrm{~cm}$, and $x>40.4 \mathrm{~cm}$ and $\sim 5 \mathrm{~cm}$ away from the iron must be small 
enough $^{2}$ not to affect the injected and extracted beams that pass by the quadrupoles.

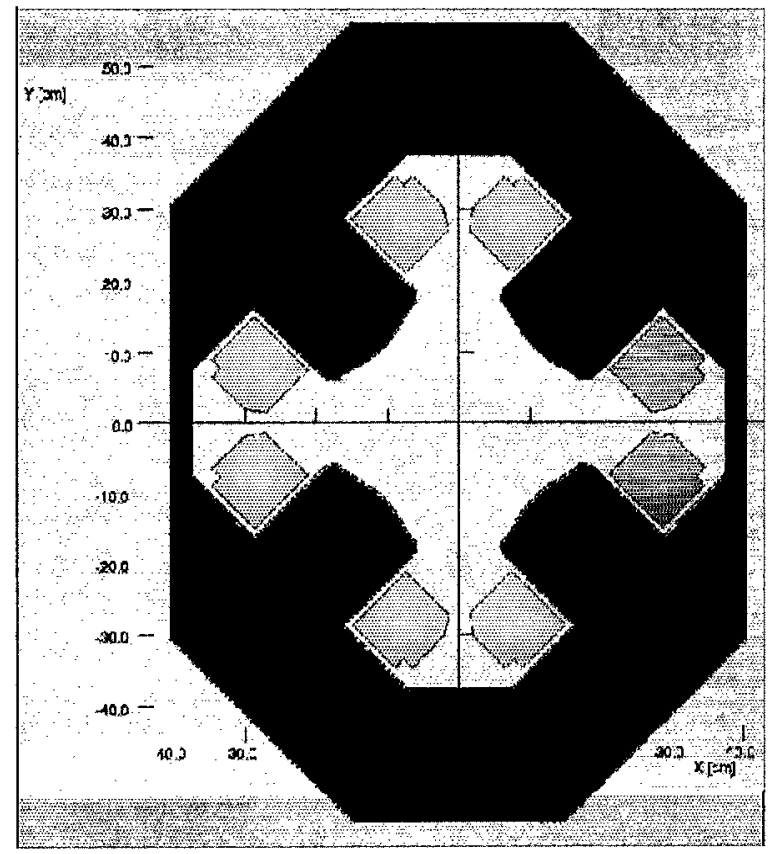

Figure 3: Cross section of the narrow quadrupole.

The B-field gradient appears in column 6 and the field non-uniformity, expressed as the ratio $B_{\text {dod }} / B_{\text {quad }}$ of the dodecapole field to the quadrupole field at a $\mathrm{r}=10 \mathrm{~cm}$ should be $\cdot 2 \times 10^{-4}$, appears in culumn 7 . This limit keeps the width of the 12pole resonance[5] from infringing into the tune spread of the beam thus allowing larger tune space for beam stability of the circulating beam.

Table 1: Requirements of the narrow quadrupoles.

\begin{tabular}{|c|c|c|c|c|c|c|}
\hline Name & $\begin{array}{c}\mathrm{R} \\
\mathrm{cm}\end{array}$ & $\begin{array}{c}\mathrm{L} \\
\mathrm{cm}\end{array}$ & $\begin{array}{c}\mathrm{W} \\
\mathrm{cm}\end{array}$ & $\begin{array}{c}\mathrm{PW} \\
\mathrm{cm}\end{array}$ & $\begin{array}{c}\mathrm{G} \\
{[\mathrm{T} / \mathrm{m}]}\end{array}$ & $\mathrm{B}_{\text {dod }} / \mathrm{B}_{\text {quad }}$ \\
\hline $30 \mathrm{Q} 44$ & 15 & 44 & 80.8 & 17.8 & $4.2,5.1$ & $\cdot 2 \times 10^{-4}$ \\
\hline $30 \mathrm{Q} 58$ & 15 & 58 & 80.8 & 17.8 & $4.2,5.1$ & $\cdot 2 \times 10^{-4}$ \\
\hline
\end{tabular}

\section{MODELLING OF THE NARROW QUAD}

The magnetic modeling of the narrow-Quad was performed to determine a) the contour of the pole face, and b) the minimum dimensions of the width and polewidth of the magnet, that satisfy the field requirements. The commercially available computer code "opera" of Vector-Fields Inc. [6] was used for the modeling of the magnet and the calculations were performed in two steps; first 2D-modeling and second 3D modeling.

\section{$3.12 D$ Modeling}

The 2D modeling establishes the contour of the polc-facc that minimizes the dodecapole multipole ${ }^{3}$. The contour of the pole face that creates the multipoles shown in Table 2

\footnotetext{
${ }^{2}$ For $1.0 \mathrm{GeV}$ operation the fringe field is $\sim 5$ Gauss

3 The symmetry of this "narrow-quad" is not four-fold symmetric therefore the dodecapole multipole is not the first allowed multipole (Table 2). However the strength of the allowed multipoles introduced
}

is very simple and is described in [8]. The 2D modeling establishes also the minimum allowed width of the ironpole $(17.8 \mathrm{~cm})$ that minimizes the magnetic saturation effects in the iron. Indeed Table 2 shows that the multipoles do not vary linearly with the quadrupole strength because of the nonlinear permeability of the iron. However the strength of the multipoles shown in Table 2, for the excitation encrgics of 1.0 and $1.3 \mathrm{GeV}$ operations is too small to affect the field quality of the magnet. The ratio $B_{\text {dod }} / B_{\text {quad }}$ at $r=10 \mathrm{~cm}$ is $\cdot 2 \times 10^{-4}$ as required.

Table 2. The strength of the allowed multipoles ${ }^{4} b_{\text {. }}$.

\begin{tabular}{|c|c|c|c|c|c|}
\hline $\begin{array}{c}\mathrm{T} \\
\mathrm{GeV}\end{array}$ & $\begin{array}{l}\mathrm{b}_{1 \text { quad }} \\
\operatorname{Tm}^{-1}\end{array}$ & $\begin{array}{l}\mathrm{b}_{3 \circ \mathrm{cos}} \\
\mathrm{Tm}^{-3}\end{array}$ & $\begin{array}{l}\mathrm{b}_{5 \text { dog }} \\
\mathrm{Tm}^{3}\end{array}$ & $\begin{array}{l}\mathrm{b}_{716 \mathrm{pgle}} \\
\mathrm{Tm}^{-}\end{array}$ & $\begin{array}{l}\mathrm{b}_{920 \mathrm{polg}} \\
\mathrm{T}^{*} \mathrm{~m}\end{array}$ \\
\hline 1.0 & 4.236 & $-1.1 \times 10^{-4}$ & 2.20 & -0.30 & $-2.6 \times 10^{5}$ \\
\hline 1.3 & 5.084 & $-2.2 \times 10^{-4}$ & -0.22 & -0.67 & $-3.1 \times 10^{5}$ \\
\hline
\end{tabular}

The absolute value of the magnetic field $\mathrm{B}_{\bmod }$ in the iron, shown in Fig.4, is below the value of $16 \mathrm{kGauss}$.

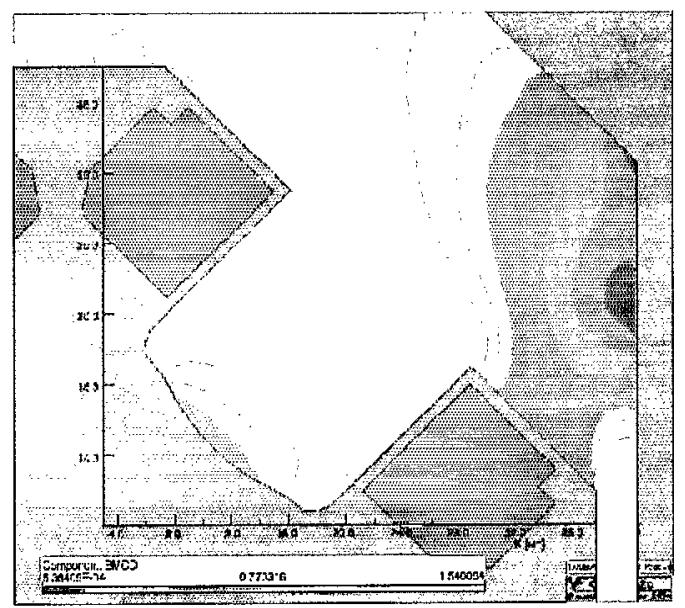

Figure 4: $\quad$ Contour plot of the $\mathrm{B}_{\text {mod }}$ in the iron.

\subsection{D Modeling}

The 3D modeling provides complete information about the magnetic field of the magnet in 3D space and helps further in the optimization of the shape of the magnet which should satisfy all the magnetic field requirements. The 3D model of a $44 \mathrm{~cm}$ long quadrupole was generated by using the same cross section as the cross section of an optimized quadrupole from the 2D calculations described earlier. The 3D magnetic field calculations have been performed using the TOSCA module of the opera computer code[6]. The aim of the 3D calculations is to minimize the relative strength ${ }^{5}$ of the allowed multipoles of the magnet by shaping the ends of the pole-pieces. The results from the $3 \mathrm{D}$ calculations of the $3 \mathrm{D}$ models were analyzed by calculating the radial field $\mathrm{B}_{\mathrm{r}}(\mathrm{r}, \mathrm{z})$ on a radius $\mathrm{r}=10 \mathrm{~cm}$, and expanding it as in the expansion (1) below: $B_{r}(r, z)=\cdot b_{n}(r, z) \cos [(n+1) \theta] \quad\{n=1$ quad $n=3$ oct $\ldots\}(1)$

due to the break of the four fold symmetry of the quad are negligible as shown in Table 2.

${ }^{4}$ The multipoles $b_{n}$ have been calculated from the expansion of the radial field $B_{r}=\Sigma b_{n} r^{n} \cos [(n+1) \theta]\{n=0$ Dip, $n=1$ Quad... $\}$ at $r=15 \mathrm{~cm}$.

${ }^{5}$ Relative to the strength of the quadrupole multipole. 
Subsequently the amplitude of the expansion coefficients that correspond to the various multipoles were compared among the various $3 \mathrm{D}$ models.

In particular, the calculated coefficient of expansion $b_{5}(r, z)$ which is a measure of the strength of the dodecapole multipole is plotted as a function of the distance $\mathrm{z}$ from the centre of the quadrupole in Fig. 5 for two 3D models a) No "pole-piece chamfer" and b) with $30.25^{\circ}$ pole-piece chamfer ${ }^{7}$ (see Fig. 6 ).

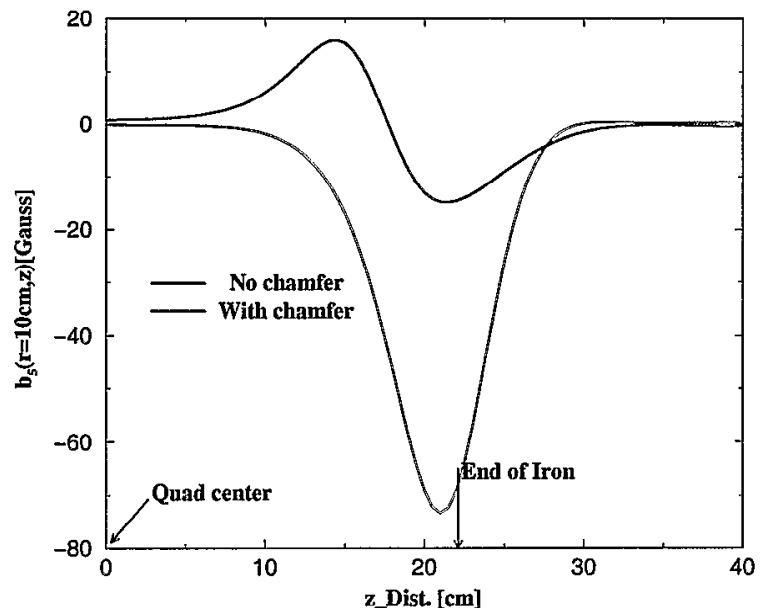

Figure 5: Plots of the expansion coefficient $b_{s}(r=10, z)$.

The variation of the $b_{5}$ coefficient plotted in Fig. 5 is due to the end effects of the magnet. In order to set a basis for comparison with the experimentally measured quantities, we calculated the values of the ratios

$R_{n}=\cdot b_{n}(r, z) d z / \cdot b_{1}(r, z) d z$ where $\cdot b_{n}(r, z) d z$ is the amplitude of the radial field $b_{n}(r, z)$ of the $n^{\text {th }}$ multipole, integrated along the z-direction at $\mathrm{r}=10 \mathrm{~cm}$.

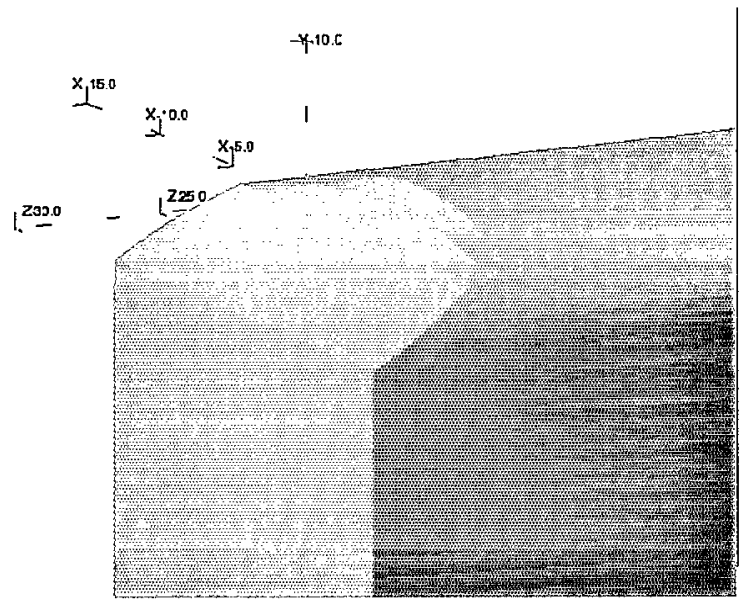

Figure 6. An isometric view of the chamfered end of one of the pole-piece of the quadrupole. A chamfer of $30.25^{\circ}$ minimizes the value of $\cdot \mathrm{b}_{5}(\mathrm{r}, \mathrm{z}) \mathrm{dz}$.

\footnotetext{
${ }^{6}$ The cross section of the pole piece of the 3D model is identical to the cross section of the 2D model.

7 The ends 'of the pole pieces have heen chamfered hy $30.25^{\circ}$. The chamfer starts at $6 \mathrm{~cm}$ from the edge of the pole face.
}

Table 3 contains the values of the calculated ratios $R_{n}$ for the various multipoles. All the value of the ratios $R_{n}$ are well below the limit of $2 \times 10^{-4}$ except the value of $R_{5}$ ratio $^{8}$ at $1.3 \mathrm{GeV}$. The value of the effective quadrupole length $\mathrm{L}_{\text {eff }}$ of the magnet is shown in column 5 for 1.0 and 1.3 GeV excitations.

Table 3: The Ratio $R_{n}$ of few allowed multipoles.

\begin{tabular}{|c|l|c|c|c|}
\hline $\mathrm{T} \mathrm{GeV}$ & $\mathrm{R}_{3 \text { ort }}$ & $\mathrm{R}_{\text {stlod }}$ & $\mathrm{R}_{\text {7lopole }}$ & $\mathrm{L}_{\text {eff }}$ [cm] \\
\hline 1.0 & $-8 \times 10^{-6}$ & $6 \times 10^{-5}$ & $-2 \times 10^{-7}$ & 53.3 \\
\hline 1.3 & $-4 \times 10^{-5}$ & $5 \times 10^{-4}$ & $-2 \times 10^{-6}$ & 53.1 \\
\hline
\end{tabular}

\section{MECHANICAL TOLERANCES}

The 3D modeling also provides an upper limit in the mechanical tolerances within which the magnet still satisfies the field requirements, thus avoiding "over or under"-design of the magnet. The mechanical tolerances were studied in a full 3D model by introducing possible mechanical errors in the optimized 3D model.

Among the possible mechanical errors studied we report:

a) An error of $0.5 \mathrm{~mm}$ in the longitudinal length of one of the poles of the magnet.

b) Rotation of a single pole-face by $1.5 \mathrm{mrad}$ about an axis parallel to the symmetry axis of the quadupole and passing through the pole tip.

In both cases (a) and (b) above the variation of the ratio $R_{5}$ was $\sim 10 \%$ of the value shown in Table 3 , with the rest of the ratios maintaining still small values as those in Table 3 .

\section{CONCLUSIONS}

Magnctic ficld calculations on 2D and 3D models of a large aperture narrow quadrupole provided optimum pole shape and dimensions for an SNS ring quadrupole which satisfies the field requirements of the SNS ring.

Upper limits on the mechanical tolerances were obtained by introducing mechanical errors on a full 3D-model.

\section{REFERENCES}

[1] J. Wei, et. al. Phys. Rev. ST AB 3, 080101 (2000)

[2] J. Wei, et. al. EPAC2000, p. 2560 Vienna, Austria

[3] N. Tsoupas et al. EPAC2000 p. 2270 Vienna, Austria

[4] Catalan-Lasheras et al. Phys. Rev. ST-AB 4, 010101

[5] A. Fedotov et al. EPAC2000 p. 1492 Vienna, Austria

[6] Vector-Fields-Inc.

[7] D. Abell, et al. EPAC2000 p. 2107 Vienna, Austria

[8] N. Tsoupas et al. BNL SNS Tech. Note 110

\footnotetext{
${ }^{8}$ Calculations show that an alternative model with a ratio $R_{5} \sim 2 \times 10^{4}$ at $1.0 \mathrm{GeV}$ generates a ratio $R_{3} \sim 3 \times 10^{4}$ at $1.3 \mathrm{GeV}$ excitation. However we have chosen to use the model that generates the $R_{n}$ shown in Table 3 .
} 